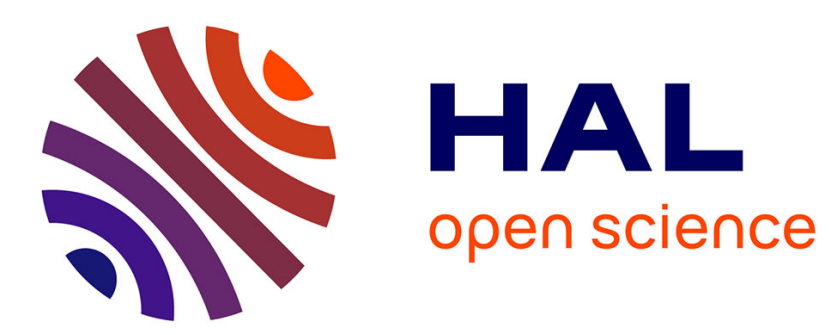

\title{
Laser solide à fonctionnement continu à la température ambiante
}

\author{
O. Deutschbein, G. Grimouille, C. Pautrat, G. Petit-Le-Du
}

\section{To cite this version:}

O. Deutschbein, G. Grimouille, C. Pautrat, G. Petit-Le-Du. Laser solide à fonctionnement continu à la température ambiante. Revue de Physique Appliquée, 1966, 1 (2), pp.128-132. 10.1051/rphysap:0196600102012800 . jpa-00242697

\section{HAL Id: jpa-00242697 https://hal.science/jpa-00242697}

Submitted on 1 Jan 1966

HAL is a multi-disciplinary open access archive for the deposit and dissemination of scientific research documents, whether they are published or not. The documents may come from teaching and research institutions in France or abroad, or from public or private research centers.
L'archive ouverte pluridisciplinaire HAL, est destinée au dépôt et à la diffusion de documents scientifiques de niveau recherche, publiés ou non, émanant des établissements d'enseignement et de recherche français ou étrangers, des laboratoires publics ou privés. 


\title{
LASER SOLIDE A FONCTIONNEMENT CONTINU A LA TEMPÉRATURE AMBIANTE
}

\author{
Par O. DEUTSChBEIN, G. GRIMOUIlle, C. PAUTRAT et G. PETIT-LE DU, \\ Département Physique, Chimie, Métallurgie, \\ Centre National d'Études des Télécommunications.
}

\begin{abstract}
Résumé. - On décrit une méthode de synthèse des monocristaux de tungstate de calcium dopé au néodyme (concentration : 0,5 à $2 \%$ ) : fusion en creuset d'iridium, sous balayage d'argon humide, par chauffage haute fréquence. Les cristaux sont tirés suivant l'axe $a$, à la vitesse de $11,5 \mathrm{~mm} /$ heure. Ils subissent ensuite un recuit de 15 heures à $1300{ }^{\circ} \mathrm{C}$.

Les barreaux découpés dans ces cristaux ont présenté l'effet laser en continu à la température ordinaire, dans une installation dont les principales caractéristiques sont indiquées. Les seuils de fonctionnement sont de 420 watts, et 900 watts, la lampe de pompage étant alimentée en courant alternatif et continu respectivement. La puissance émise, pour une puissance de pompage de $1 \mathrm{kwatt}$, dépasse 0,5 watt.
\end{abstract}

Abstract. - A method of synthesis of neodymium doped calcium tungstate single crystals is described ( $\mathrm{Nd}$ concentration from 0.5 to 2 per cent) : by melting in iridium crucibles, with moist argon circulation, and high frequency heating.

Cristals are pulled with $a$-axis orientation, with a speed of growth of $11,5 \mathrm{~mm} / \mathrm{hour}$, and are annealed during 15 hours at $1300^{\circ} \mathrm{C}$.

Rods, cut from these crystals, have shown continuous laser operation at room temperature with an apparatus described here. The thresholds are 420 and 900 watts, the pumping lamp being supplied with a. c. and d. c. respectively. The output power, for one kilowatt input power, is more than half a watt.

I. Introduction : Choix du matériau. - Pour les applications du íaser aux télécommunications, il nous a semblé intéressant d'étudier un dispositii susceptible de produire une émission continue laser d'environ 1 watt, fonctionnant à la température ambiante et ne récessitant pas de gaz liquéfiés.

Les dispositifs lasers à fonctionnement continu à la température ambiante peuvent être réalisés actuellement avec deux matériaux assez différents : laser à gaz; laser à solides fluorescents.

Les lasers à gaz (mélange $\mathrm{He}-\mathrm{Ne}$ ) ont été réalisés les premiers en fonctionnement continu [1]. Ils sont caractérisés par une très faible concentration en atomes actifs (de l'ordre de $10^{16}$ atomes $/ \mathrm{cm}^{3}$ ). De ce fait l'indice de réfraction est très proche de l'unité : 1,000 003 environ : ainsi, les variations de cet indice, dues aux fluctuations de température ou d'homogénéité du gaz, sont très faibles. En conséquence, la qualité de l'émission du point de vue de la monochromaticité, de la stabilité de fréquence et de la divergence du faisceau est très bonne, mais d'autre part, le gain par unité de longueur est faible, ainsi que la puissance émise : celle-ci est de l'ordre de quelques dizaines de milliwatt pour des longueurs de l'ordre de $50 \mathrm{~cm}$, il faut donc utiliser des cavités de quelques mètres de longueur pour obtenir des puissances supérieures au watt, éventuellement avec un bon rendement pour les lasers à gaz moléculaire.

Par contre, dans les lasers à solides fluorescents, la concentration en atomes actifs est environ $10^{4}$ fois plus grande, de l'ordre de $10^{-0}$ atomes $/ \mathrm{cm}^{3}$, il en résulte un gain très élevé par unité de longueur : les cavités de $5 \mathrm{~cm}$ de longueur peuvent donc émettre des puissances de l'ordre de 1 watt. De plus, les lasers à solides fluorescents sont suceptibles d'un pompage direct par le soleil, caractéristique éventuellement intéressante pour des applications spatiales ou de télécommunications. En contrepartie, ces substances possèdent un indice de réfraction de l'ordre de 1,5 à 2 et, de ce fait, les variations éventuelles de l'homogénéité et de la température peuvent être gênantes. C'est pourquoi un soin particulier doit être pris lors de la fabrication du matériau, ainsi que dans le montage du barreau dans l'équipement laser. Pour obtenir un échauffement minimum par le rayonnement de la source de pompage, on doit choisir une substance avec un rendement optimum de pompage. Pour le tonctionnement à la température ambiante, on doit utiliser un système énergétique à quatre niveaux de telle façon que le niveau final de la transition laser soit pratiquement inoccupé à la température ambiante. D'après la loi de Boltzmann, ce niveau doit être situé à au moins $0,25 \mathrm{eV}$, ou exprimé en nombre d'onde, à au moins $2000 \mathrm{~cm}^{-1}$ au-dessus du niveau fondamental. Parmi les terres rares, utilisées comme dopant dans les solides fluorescents, l'ion trivalent du néodyme $\left(\mathrm{Nd}^{+++}\right)$possède un système quantique qui remplit bien cette condition (fig. 1). De plus, cet ion possède plusieurs bandes d'absorption 
fortes, situées dans la partie visible et proche infrarouge du spectre, ce qui rend assez efficace le pompage par des lampes fabriquées industriellement (lampes à arc de mercure, de xénon ou lampes à filaments de tungstène dans une atmosphère d'iode). Dans la figure 1, sont indiquées par des flèches fines, les transitions d'absorption servant au pompage et, par des grosses flèches, les transitions laser, dont la principale est à $1,06 \mu$.

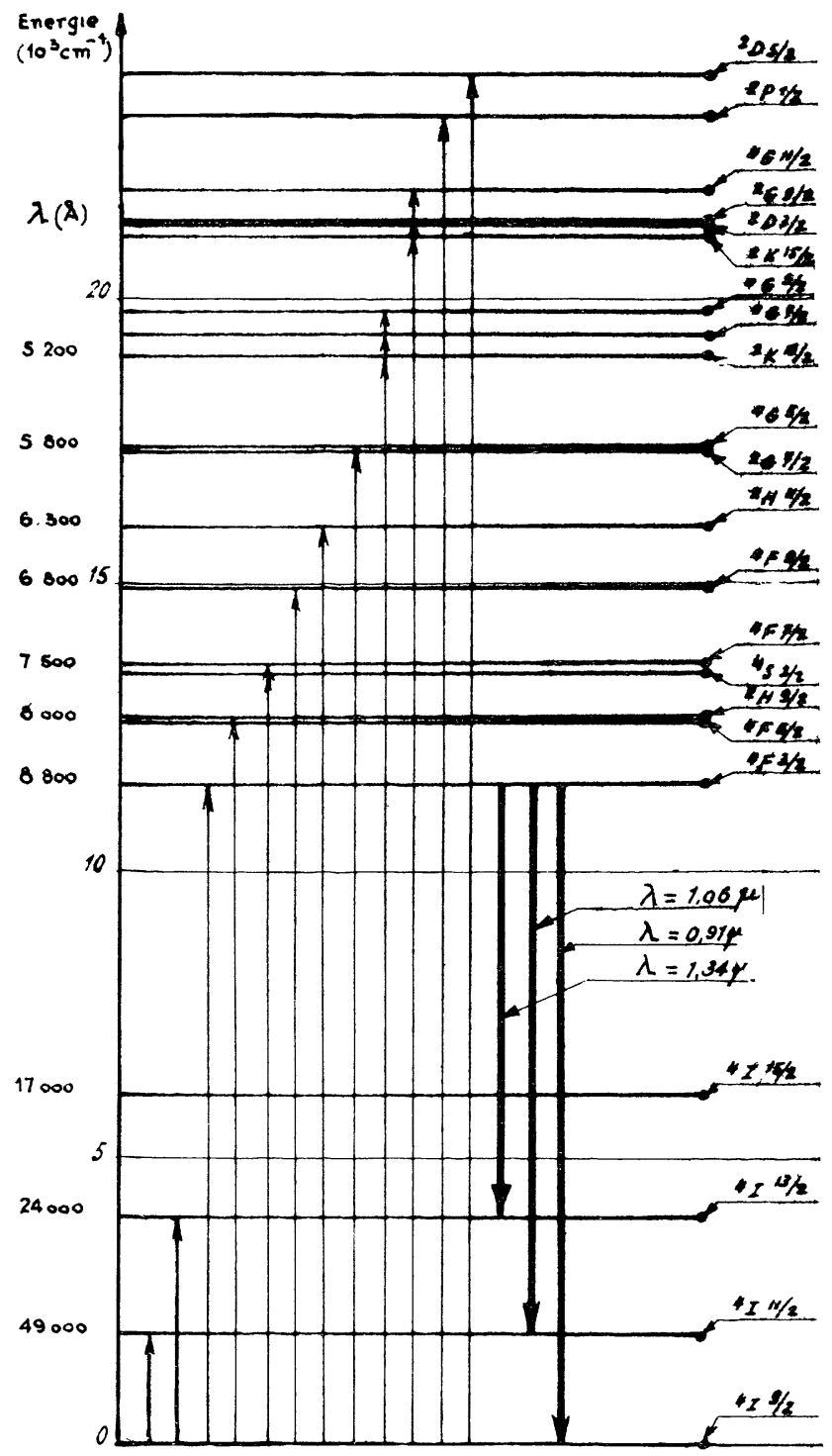

FIg. 1. - Niveaux d'énergie de l'ion $\mathrm{Nd}^{3+}$ isolé. $\lambda=1,06 \mu$ : transition laser principale

$\lambda=0,91 \mu\}$ Transitions laser observées avec des

$\lambda=1,34 \mu\} \quad$ réflecteurs sélectifs.

Pour être fluorescents, les ions de néodyme doivent être incorporés dans une matrice solide incolore (cristal ou verre) qui, en raison des champs électriques de ses constituants, modifie les niveaux d'énergie représentés à la figure 1, par dédoublement de ces niveaux suivant la symétrie de ces champs. En même temps, la symétrie des champs cristallins agit sur les probabilités des transitions entre les différents niveaux, transitions qui, en absence de champ électrique, seraient interdites.

Après des essais infructueux avec plusieurs fluorures : $\mathrm{CaF}_{2}, \mathrm{SrF}_{2}, \mathrm{LaF}_{3}$, nous avons finalement choisi le tungstate de calcium $\mathrm{CaWO}_{4}$, pour les raisons suivantes :

- le spectre d'émission est constitué de raies étroites ce qui donne un gain élevé d'amplification en effet laser ;

- la température de fusion de cette ${ }^{-}$substance $\left(1620^{\circ} \mathrm{C}\right.$ ) est du même ordre que celle du silicium et, de ce fait, l'expérience acquise dans la synthèse des monocristaux de silicium est facilement exploitable ;

- le fonctionnement en effet laser continu a été obtenu à la température ambiante, par ailleurs [2], bien qu'avec des seuils d'excitation plus élevés.

\section{Préparation des monocristaux de tungstate de} calcium. - II.1. MÉth ode. - La méthode retenue est celle de Czochralski, fréquemment utilisée dans les laboratoires comme dans lindustrie et abondamment décrite dans la littérature surtout à propos des semiconducteurs. Plusieurs études ont déjà été, dans ce domaine, consacrées au $\mathrm{CaWO}_{4}[3,4]$. Nous décrirons comment nous avons appliqué au $\mathrm{CaWO}_{4}$ une technique d'élaboration de monocristaux connue.

II.2. Matière première. - L'analyse spectrographique de $\mathrm{CaWO}_{4}$ en poudre (qualité " optique ") de différentes origines a donné les résultats suivants : les produits commercialisés contiennent en général des traces importantes de $\mathrm{Mg}, \mathrm{Al}$, Si et, éventuellement à l'état de traces faibles, du $\mathrm{Cu}, \mathrm{Fe}$ et $\mathrm{Sr}$. Les mêmes analyses ont montré que, grâce à la ségrégation des impuretés, l'opération de tirage purifie considérablement le matériau, à l'exception près qu'il peut y avoir une contamination du cristal par le creuset ; celle-ci est beaucoup moins importante avec les creusets en iridium qu'avec ceux en rhodium [5]. L'effet de ségrégation permet d'effectuer une purification ultime du $\mathrm{CaWO}_{4}$ par un premier tirage à partir de la poudre fondue : pour chaque monocristal, seule une moitié est conservée en vue du tirage définitif. un quart en étant systématiquement éliminé à chaque extrémité. La contamination du bain par le creuset, que nous avons évaluée par une méthode radiochimique dans le cas de creuset en iridium, augmente en fonction du vieillissement $\mathrm{du}$ creuset, vraisemblablement en raison d'une modification de la structure superficielle de celui-ci.

II.3. Installation de tirage. - Les figures 2 et 3 donnent toutes les indications sur la disposition 


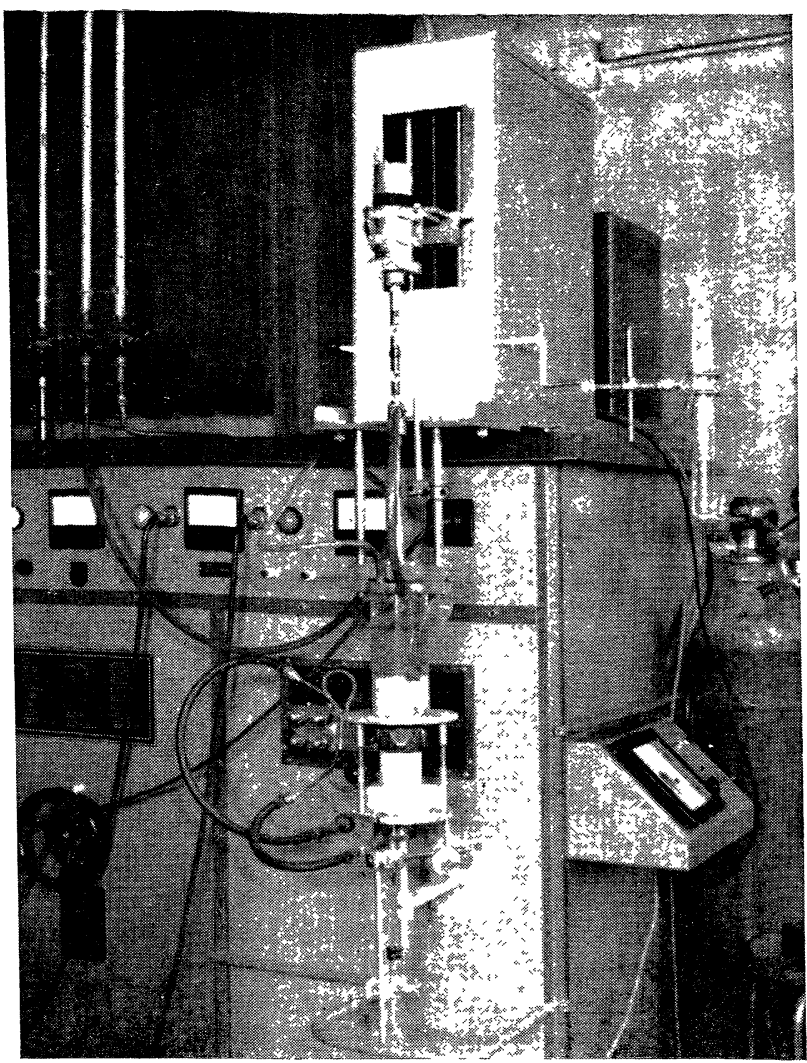

FIG. 2. - Vue d'ensemble de l'installation de tirage.

de l'appareil de tirage et sur les matériaux qui le composent.

Le chauffage est obtenu par induction dir scte dans le creuset en rhodium ou en iridium à partir d'une spire unique alimentée par un générateur $\mathrm{H}$. F. de tréquence $1 \mathrm{MHz}$ et de puissance $12 \mathrm{~kW}$. La régulation de température est assurée à $\pm 2{ }^{\circ} \mathrm{C}$ par un dispositif électronique. L'enceinte est balayée de haut en bas par un courant d'argon d'environ $1 \mathrm{l} / \mathrm{mn}$. Le gaz est humidifié par barbotage dans l'eau, elle-même purifiée par distillation et par passage dans une colonne à résine échangeuse d'ions.

La tige porte-germe est refroidie par un courant d'eau. Le germe, de section carrée $3 \times 3 \mathrm{~mm}$, est serré par un fil de platine rhodié sur une équerre de même alliage fixée à l'extrémité de la tige.

II.4. Mode opératoire. - Le creuset est garni de $180 \mathrm{~g}$ de $\mathrm{CaWO}_{4}$ purifié par des tirages préliminaires comme indiqué au paragraphe II.2. On y ajoute l'élément de dopage sous forme de $\mathrm{Na} \mathrm{Nd}\left(\mathrm{WO}_{4}\right)_{2}$, également purifié au préalable par tirage, ainsi qu'un léger excès de $\mathrm{Na}_{2} \mathrm{WO}_{4}$ destiné à compenser la perte en sodium de 20 à $30 \%$ due à l'évaporation. La quantité de dopant est calculée en tenant compte du coefficient de ségrégation du

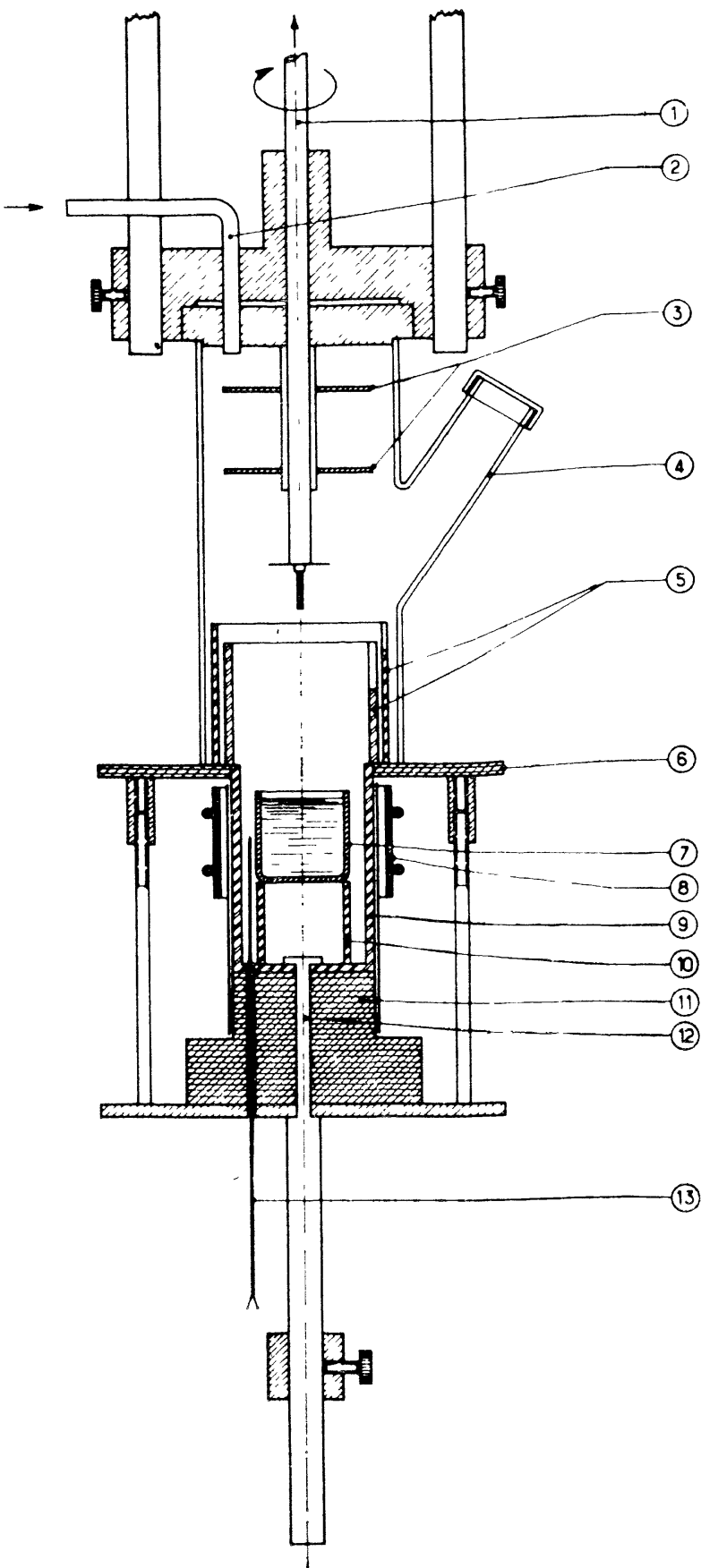

Fig. 3. - Schéma de l'installation de tirage. (1) Tige porte germe refroidie à l'eau. (2) Arrivée A humide. (3) Réflecteurs en acier inox. (4) Manchon en verre de silice avec lunette de visée. (5) Écrans en alumine. (6) Couronne de silice. (7) Creuset iridium. (8) Inducteur. (9) Creuset en alumine. (10) Support de creuset en alumine. (11) Brique réfractaire. (12) Évacuation des gaz. (13) Thermocouple $\mathrm{Pt}-\mathrm{Pt} 10 \% \mathrm{Rh}$. Échelle 1/4.

néodyme, déterminé empiriquement à 0,45 dans nos conditions de travail. 
Une fois le creuset mis en place dans l'enceinte, le balayage d'argon humide est effectué durant 15 minutes environ. Puis, le creuset est chauffé jusqu'au point de fusion de son contenu $\left(\simeq 1620^{\circ} \mathrm{C}\right)$ et le tirage est opéré suivant le procédé classique, les vitesses de translation et de rotation du cristal étant respectivement de $11,5 \mathrm{~mm} / \mathrm{h}$ et 120 tours $/ \mathrm{mn}$.

II.5. Recuit. - Tous les cristaux subissent ensuite un recuit en atmosphère d'oxygène. Le cycle de recuit est le suivant: montée depuis la tempésature ambiante jusqu'à $1300{ }^{\circ} \mathrm{C}$ en $9 \mathrm{~h}$, palier durant $15 \mathrm{~h}$, puis refroidissement de $50{ }^{\circ} \mathrm{C} / \mathrm{h}$. Ce traitement, qui ne semble pas améliorer sensiblement les caractéristiques optiques des cristaux, est indispensable, d'une part pour éliminer les tensions d'origine thermique qui les rendent fragiles au découpage, d'autre part pour éviter leur déformation lors de leur utilisation en fonctionnement laser.

iI.6. Cristaux obtenus. - Le tirage s'effectue suivant la direction de l'axe $a$, l'orientation $c$ produisant des cristaux de moins bonne qualité optique et de plus grande fragilité.

Le diamètre des cristaux peut varier de 8 à $15 \mathrm{~mm}$ (fig. 4) sans qu'on observe une altération de leur qualité. Un diamètre supérieur conduit à une augmentation de la fragilité. Leur longueur est d'environ 60 à $65 \mathrm{~mm}$.

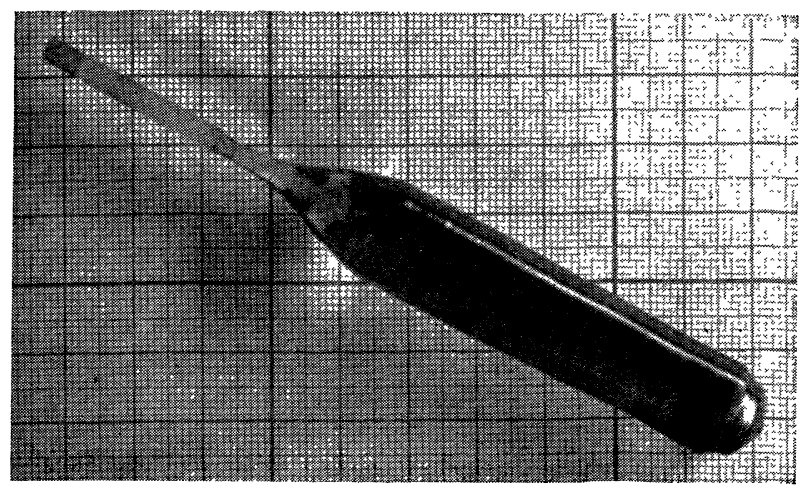

Fig. 4. - Monocristal de $\mathrm{CaWO}_{4}$ et son germe.

Les cristaux sont généralement exempts de défauts visibles à l'œil nu, mais on note parfois, l'existence de " nuages " intérieurs [3] qui peuvent apparaître pour les raisons suivantes :

- mauvaise qualité de la matière première ;

- vitesse de tirage trop grande ;

- diamètre du cristal trop grand par rapport à celui du creuset;

- variation de température du bain ;

- excès de dopant $(>2 \%)$.

Les essais de dopage ont porté sur des concentrations atomiques $\mathrm{Nd} / \mathrm{Ca}$ variant de 0,5 à $2 \%$.
Les seuils les plus bas ont été obtenus sur des échantillons contenant de 0,5 à $1 \%$ de $\mathrm{Nd}$.

II.7. Mise en forme. - Les cristaux sont, dans une première phase, découpés à la scie diamantée en bâtonnets de section carrée $4 \times 4 \mathrm{~mm}$ et de longueur $50 \mathrm{~mm}$. Ces bâtonnets sont rectifiés à la meule diamantée et transformés en cylindres de $3 \mathrm{~mm}$ de diamètre dont la surface latérale est polie à la poudre d'alumine ou de diamant, de même que les extrémités, taillées en calotte sphérique de rayon de courbure $50 \mathrm{~mm}$. Ces extrémités sont soit argentées, l'une de ces argentures étant légèrement transparente $(2$ à $5 \%)$, soit revêtues de couches multidiélectriques.

III. Réalisation d'un laser à fonctionnement continu. - Pour vérifier la qualité des barreaux, le seuil de fonctionnement laser en impulsion est mesuré dans les conditions suivantes : le barreau est placé contre un tube à éclair rectiligne ; une feuille d'argent poli, servant de réflecteur, est enroulée autour de l'ensemble: les seuils typiques obtenus dans ces conditions sont de l'ordre de 0,3 à 0,5 joule, à température ambiante.

Pour le fonctionnement continu, l'installation de pompage est essentiellement constituée par un miroir cylindrique à section elliptique, fermé par deux miroirs plans, munis de deux ouvertures afin de pouvoir placer aux lignes focales du miroir cylindrique la lampe d'excitation et le barreau laser. Le miroir cylindrique est formé de deux demicoquilles usinées mécaniquement. Pour augmenter le coefficient de réflexion de ce miroir, celui-ci est recouvert intérieurement d'une couche d'argent. La lampe d'excitation utilisée est une lampe à arc au xéron, l'arc ayant une longueur de $50 \mathrm{~mm}$. La puissance nominale d'alimentation de cette lampe est de 1 kilowatt alternatif.

Le cliché 5 montre une vue générale de l'installation, la figure 6 représente la cavité de concentration (un demi-miroir plan étant enlevé).

La lampe et le barreau sont maintenus à la température ambiante par une circulation commune d'eau courante. Des mesures directes ont montré que le centre du barreau s'échauffe jusqu'à une température d'environ $100^{\circ} \mathrm{C}$, dans ces conditions.

Une puissance électrique de 420 watts alternatifs est suffisante pour obtenir l'effet laser. Le fonctionnement totalement continu a été réalisé avec une puissance électrique de 900 watts : cette valeur plus élevée est due au fait que la lampe utilisée actuellement n'est pas prévue pour un fonctionnement en courant continu.

La puissance moyenne de l'émission laser à $1,06 \mu$ dépasse 0,5 watt, pour une puissance de pompage de 1 kilowatt; une puissance de sortie plus importante sera obtenue avec des miroirs multidiélectriques de transmissions optimales. 


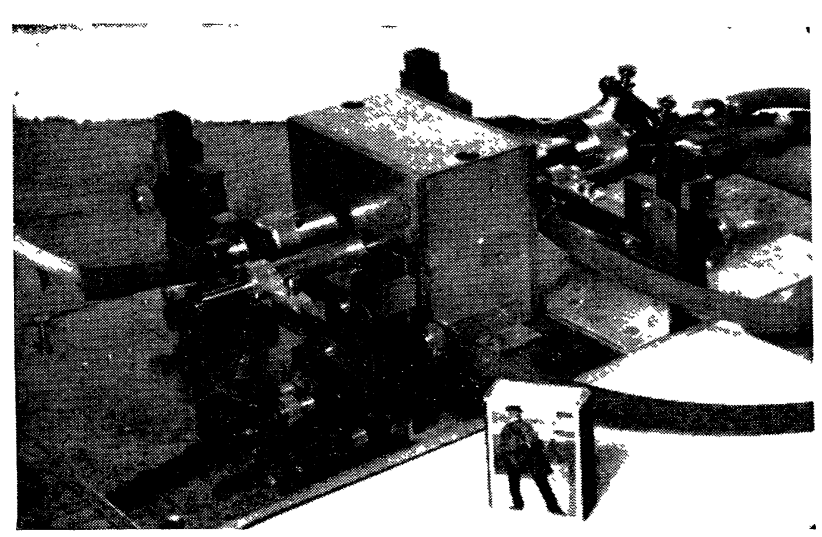

FIG. 5. - Vue générale de l'installation laser à fonctionnement permanent.

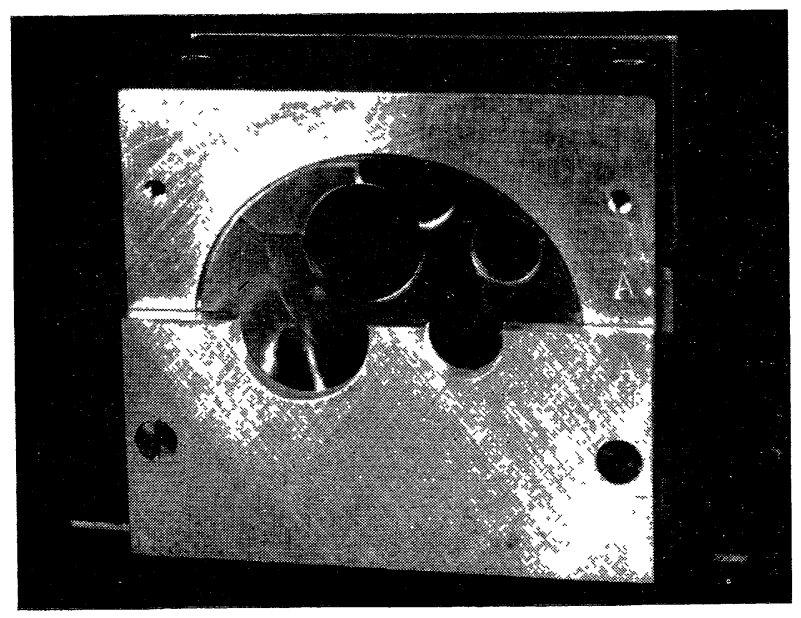

FIG. 6. - Cavité de concentration optique.

Les cristaux résistent parfaitement aux conditions de fonctionnement laser : des essais, à la puissance maximum de pompage (1 kilowatt), d'une durée supérieure à une heure, n'ont affecté ni le seuil ni la puissance de sortie émise.

L'émission peut être visualisée au moyen d'un convertisseur d'image infrarouge où l'on aperçoit la structure en " modes " ( fig. 7), caractéristique de l'émission laser. La variation de l'émission en fonc- tion du temps peut être examinée sur un oscilloscope à l'entrée duquel on envoie le signal fourni par une cellule photoélectrique.

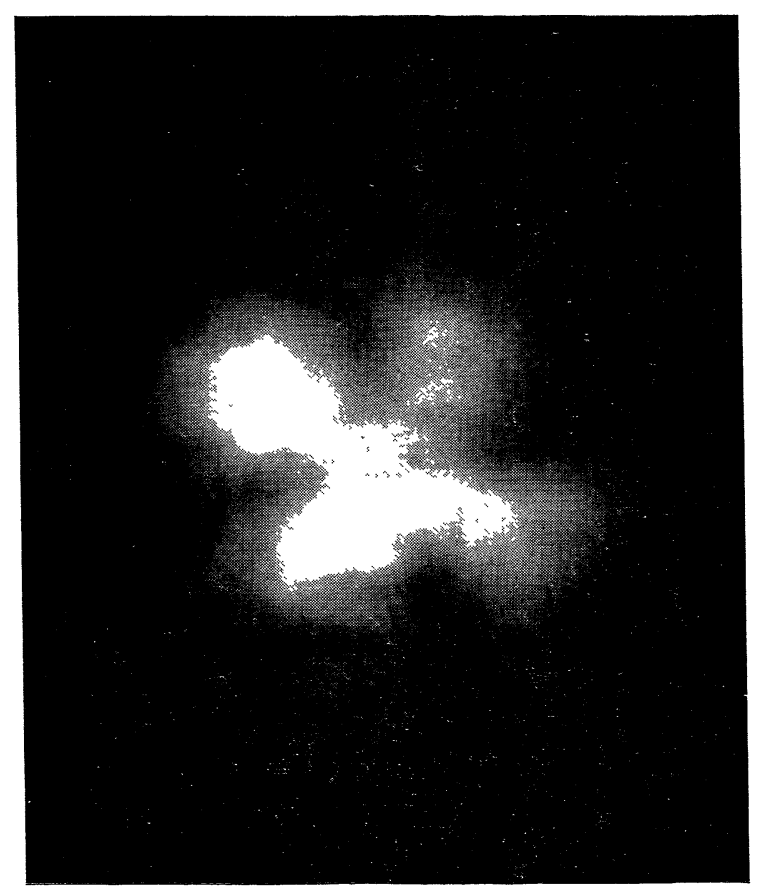

Fig. 7. - Structure en modes de la tache laser.

Au scuil, une fraction notable de l'émission est constituée d'impulsions de relaxation superposées à un signal continu, mais dès que la puissance de pompage augmente, la part relative de ces impulsions diminue très rapidement.

IV. Conclusions. - Un tel dispositif, qui présente l'avantage d'être peu encombrant et de ne pas nécessiter de gaz liquéfiés, fournit une émission continue laser puissante pouvant être utilisée pour des buts scientifiques ou de télécommunications. Les caractéristiques actuelles seront vraisemblablement améliorées rapidement par une meilleure élaboration des cristaux et des miroirs multidiélectriques, et surtout par une amélioration de la cavité cylindrique elliptique.

Manuscrit reçu le 7 avril 1966.

\section{BIBLIOGRAPHIE}

[1] Javan (A.) et autres, Phys. Rev. Letters, 1961, 6, 106

[2] Johnson (L. F.), J. Appl. Physics, avril 1963 vol. 34, part. 1, 897-909.

Gurs (K.), J. Appl. Math. Phys., 1965, 16, 49-62.

[3] Nassau (K.) et Broyer (A. M.), Calcium Tungstate : Czochralski Growth, Perfection and Substitution J. Appl. Physics, 1962, 33, no 10, 3064.
[4] Brissot (J. J.) et Raynaud (H.), Préparation et propriétés des monocristaux de $\mathrm{WO}_{4} \mathrm{Ca}$ destiné à des masers optiques. J. Physique (Physique Appliquée), 1964, 25, no 11, 199 A.

[5] Shappirio (R.), Aucoin (T. R.) et Gualtieri (J.G.), Metallic Scattering Centers in $\mathrm{CaWO}_{4}$ Laser Crystals. J. Appl. Physics, 1963, 34, no 12, 3643. 